\title{
Medium dose ultraviolet A1 phototherapy and mRNA expression of interleukin 8, interferon $\gamma$, and chemokine receptor 4 in acute skin lesions in atopic dermatitis
}

\author{
Jarosław Bogaczewicz ${ }^{1}$, Karolina Malinowska ${ }^{1}$, Anna Sysa-Jedrzejowska², Anna Wozniacka ${ }^{1}$ \\ ${ }^{1}$ Department of Dermatology and Venereology, Medical University of Lodz, Lodz, Poland \\ ${ }^{2}$ Department of Social Sciences, University of Social Sciences, Lodz, Poland
}

Adv Dermatol Allergol 2016; XXXIII (3): 170-175

DOI: 10.5114 /ada.2016.60609

\begin{abstract}
Introduction: Mechanisms responsible for UVA1 efficacy in atopic dermatitis (AD) are not fully elucidated. Aim: To investigate IL-8, CCR-4, and IFN- $\gamma$ mRNA expression in AD before and after UVA1, to identify correlations among them, and to determine whether and to what degree mRNA expression is influenced by UVA1.

Material and methods: Twenty-five patients with AD underwent medium dose UVA1-phototherapy at daily dosages of $10,20,30,45$, and then continuing $45 \mathrm{~J} / \mathrm{cm}^{2}$ up to 20 days, from Monday to Friday for 4 weeks. Before and after UVA1, biopsies from acute skin lesions were studied using reverse-transcription and RT-PCR.

Results: The levels of CCR-4 mRNA correlated with those of IFN- $\gamma$, both before and after UVA1 phototherapy $(p<0.05)$. A significant correlation was found after UVA1 between mRNA levels of IL-8 and IFN- $\gamma(p<0.05)$. After UVA1 an increase in IL-8 mRNA expression in comparison to the baseline assessment $(p=0.02)$ was found, while no significant difference was revealed in the expression of CCR-4 and IFN- $\gamma$ mRNA. UVA1 improved both SCORAD and severity of $A D(p<0.001)$. SCORAD and the severity of AD did not correlate with the degree of expression of measured cytokine mRNA, neither before nor after UVA1.

Conclusions: CCR- 4 is expressed in parallel with IFN- $\gamma$ in acute skin lesions of patients with AD both before and after UVA1 phototherapy. UVA1 significantly improves SCORAD index, lessens the severity of AD and increases the expression of IL-8, with no direct effects on other studied molecules.
\end{abstract}

Key words: atopic dermatitis, phototherapy, UVA, interferon, interleukin.

\section{Introduction}

Phototherapy is usually a part of the total treatment plan for the management of patients with atopic dermatitis (AD) [1, 2]. Ultraviolet A1 (UVA1) is effective in managing flares, whereas narrow-band UVB has been recommended for chronic moderate forms of the disease [2]. The therapeutic effectiveness of UVA1 in AD was found to accompany a decrease in the serum level of eosinophil cationic protein, with reductions in the numbers of dermal Langerhans cells and mast cells, as well as relative numbers of intraepidermal lgE+ Langerhans cells, increase in the percentage of dermal CD8+ cells and a reduction in interleukin (IL)-5, IL-13 and IL-31 mRNA expression [3-6]. Twenty years ago, Kimata and
Lindley found that plasma IL-8 concentrations decreased as AD improved during treatment [7], and more recently, Kreuter et al. [8] noted a decrease in IL-8 mRNA occurring as a response to UVA1 phototherapy in localized scleroderma. Interleukin-8 is an important mediator of chemotaxis [9]. In a recent study, IL-8 content in the stratum corneum was found to be an indicator of the severity of inflammation in AD lesions [10]. It is now known that a range of cells, including keratinocytes, mast cells and dendritic cells, are capable of secreting IL-8 when exposed to inflammatory stimuli $[9,11-18]$. Human keratinocytes were found to express IL-8 after stimulation with heat-killed Staphylococci, suggesting that bacterial load on the skin surface elicits inflammation, which begs the

Address for correspondence: Jarosław Bogaczewicz MD, PhD, Department of Dermatology and Venereology, Medical University of Lodz, 1 Hallera St, 91-647 Lodz, Poland, phone: +48 4263930 92, e-mail: jaroslaw.bogaczewicz@umed.lodz.pl Received: 17.09.2014, accepted: 28.02.2015. 
question of whether clinical efficacy of UVA1 irradiation is linked with changes in the expression of IL-8 and other cytokines involved in the pathogenetic pathways of AD, such as chemokine receptor 4 (CCR4), which is important in the recognition of skin vasculature by circulating T cells and in directing lymphocytes $[14,15]$. In addition, recent data indicate that skin-infiltrating $T$ cells are capable of producing interferon (IFN)- $\gamma$, especially in the chronic phase of the disease, indicating that pathogenesis of AD is not limited to a type-2 cytokine pattern [16].

\section{Aim}

Therefore, the aim of the study was to investigate the expression of IL-8, IFN- $\gamma$, and CCR4 mRNA in specimens of acute skin lesions of patients with $A D$ before and after UVA1 therapy and to identify any correlations which might exist among them. Additionally, the study examines whether UVA1 irradiation influences the expression of these mediators and whether this expression corresponds with the clinical efficacy of UVA1.

\section{Material and methods}

\section{Eligibility criteria}

The study included a limited number of 25 patients with atopic dermatitis, including 14 males and 11 females, aged 18 to 61, mean $32.4 \pm 11.54$ years. The diagnosis was based on the classification of Hanifin and Rajka [13]. In the clinical assessment of atopic dermatitis, to help minimize the bias, only 1 person performed SCORAD index evaluation, and this evaluator was unaware of the results of the molecular studies [17].

The exclusion criteria were pregnancy, glaucoma, cataract, claustrophobia, photosensitivity, solar urticaria, administration of phototoxic or photoallergic drugs, immunosuppressants, retinoids, glucocorticoids, the use of sunbeds or other artificial UV sources during the previous 6 months and during the study. The study was approved by the local Ethics Committee (RNN/16/14/KE).

\section{UVA1 phototherapy}

A Medisun 24000 (Schulze \& Bohm GmbH, Germany) was used for medium dose UVA1 phototherapy. The protocol comprised one exposure daily, on Monday-TuesdayWednesday-Thursday-Friday, from a starting dose of $10 \mathrm{~J} / \mathrm{cm}^{2}$ rising through 20 and 30 on consecutive days to $45 \mathrm{~J} / \mathrm{cm}^{2}$. The final dose of $45 \mathrm{~J} / \mathrm{cm}^{2}$ was maintained for up to 20 consecutive days of phototherapy. Topical management was limited to the use of emollients. No systemic drugs for atopic dermatitis were administered. Biopsies were taken from acute skin lesions, which were clinically determined when erythema or edema or oozing exceeded one point according to SCORAD index. The first biopsy was taken before phototherapy and the sec- ond one within $2 \mathrm{~h}$ after the last UVA1 irradiation. Skin samples were frozen at $-70^{\circ} \mathrm{C}$ until assay.

\section{Total RNA extraction}

Total RNA was extracted from biopsied skin samples using the RNeasy mini kits (Qiagen), according to the manufacturer's protocol. In the initial step, a buffer containing $\beta$-mercaptoethanol was added to Eppendorf tubes containing frozen samples. The samples were then homogenized using a Qiagen homogenizer (Tissue Ruptor) and centrifuged for $3 \mathrm{~min}$ at $14000 \mathrm{rpm}$. RNA was quantified using a PicoDrop spectrophotometer (Picodrop Limited). The quality of RNA samples was analyzed by measuring the absorption ratio at $260 / 280 \mathrm{~nm}$. The purified total RNA was immediately used for cDNA synthesis or stored at $-80^{\circ} \mathrm{C}$.

\section{Reverse transcription}

cDNA was generated with High Capacity cDNA Reverse Transcription Kits (Applied Biosystems Inc., Foster City, CA), according to the reverse transcription protocols. The starting material comprised $2 \mu$ g of DNAse-treated total RNA, to which the following was added: $2 \times$ RT master mix containing $2 \mu$ l of $10 \times$ RT Buffer, $0.8 \mu$ l of $25 \times$ dNTP Mix (100 mM), $2 \mu$ l of $10 \times$ RT Random Primers, $1 \mu \mathrm{l}$ MultiScribe ${ }^{\mathrm{TM}}$ Reverse Transcriptase and $1 \mu \mathrm{l}$ RNase Inhibitor per $20 \mu$ reaction. Reverse transcription was performed in conditions optimized for use with the kit: $25^{\circ} \mathrm{C}$ for $10 \mathrm{~min}, 37^{\circ} \mathrm{C}$ for $120 \mathrm{~min}, 85^{\circ} \mathrm{C}$ for $5 \mathrm{~min}$. The samples were kept frozen at $-20^{\circ} \mathrm{C}$.

\section{mRNA expression}

mRNA quantification of selected genes and an endogenous control (actin beta, ACTB) was performed using standard TaqMan ${ }^{\oplus}$ Gene Expression Assays (Applied Biosystems). Hs99999903_m1 was used as endogenous controls, and Hs00174103_m1, Hs00989291_m1, and Hs00747615_s1 for the assessment of IL-8, IFN- $\gamma$, and CCR-4.

Real-time TaqMan qPCR was performed in 96-well optical plates using the HT 7900 Fast Real-Time PCR System (Applied Biosystems) and analyzed using Sequence Detection System 2.3 Software. The results of target genes were represented as $\Delta \mathrm{Ct}$, which represents the differences in cycle threshold numbers between the target gene and endogenous control.

\section{Statistical analysis}

Statistical analysis was performed using Statistica software, version 10.0 (Statsoft, Poland). Data estimation with the Shapiro-Wilk test did not confirm a Gaussian distribution of the studied parameters. To find correlations, the Spearman correlation coefficient was estimated. Wilcoxon signed-rank test was used to compare two repeated measurements in a sample of atopic dermati- 


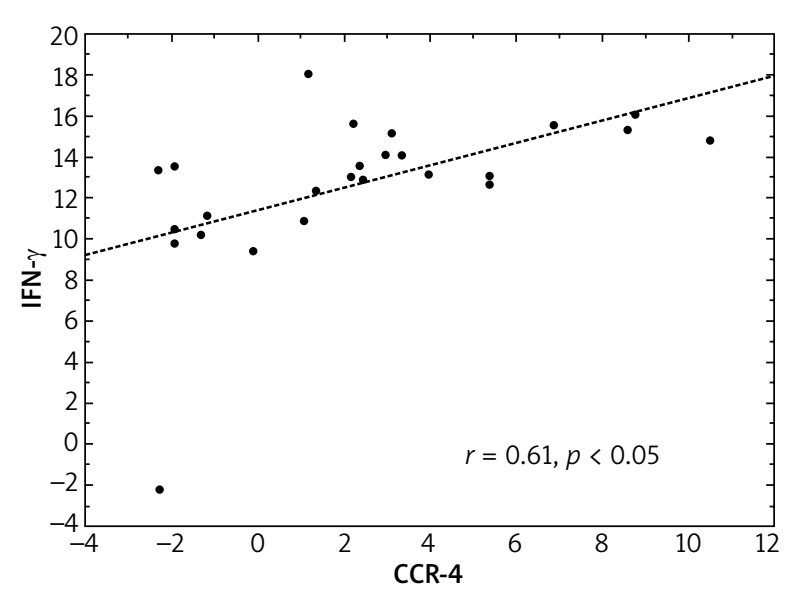

Figure 1. Correlation between mRNA levels of CCR-4 and IFN- $\gamma$ in acute skin lesions of patients with AD before UVA1 phototherapy

A
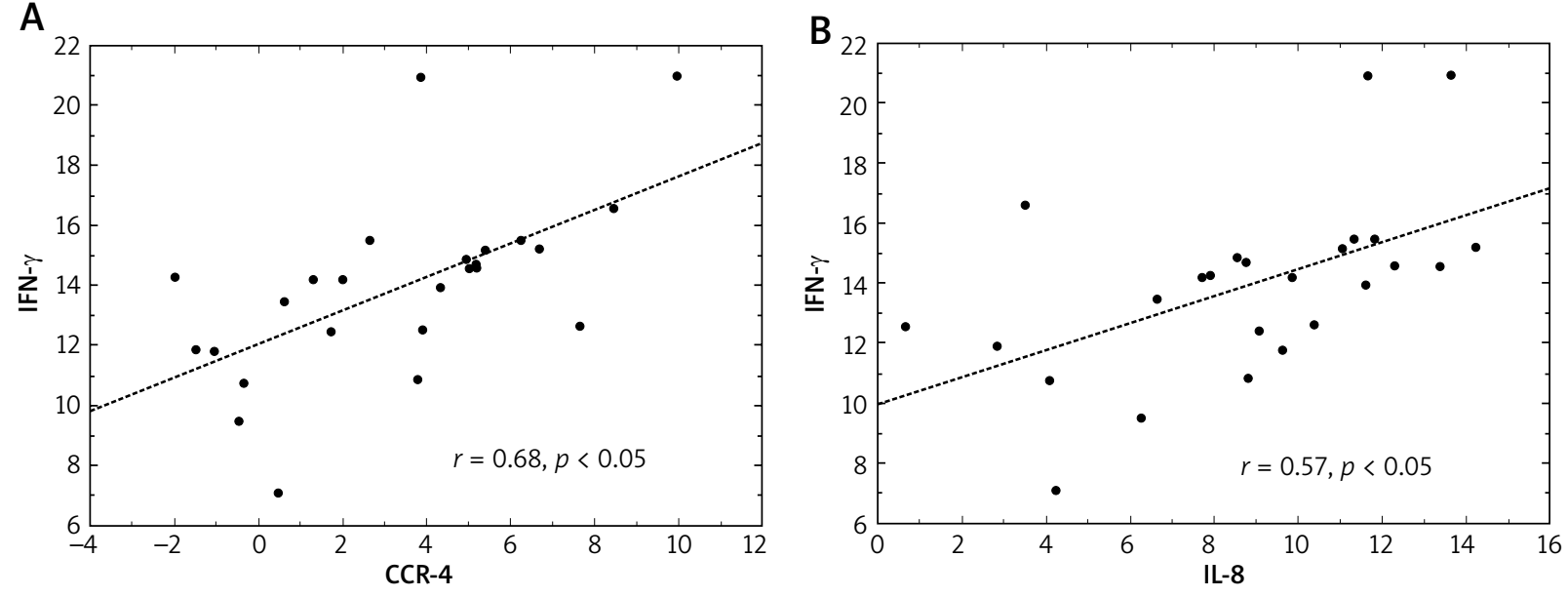

C

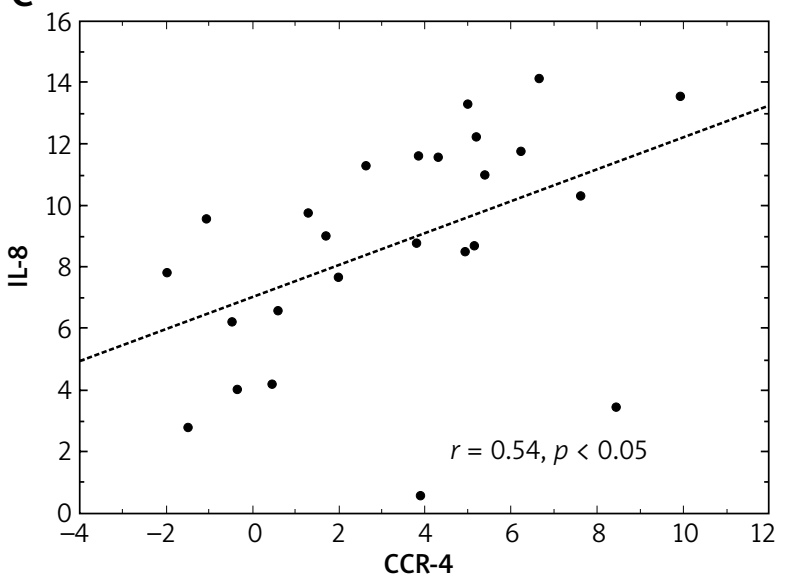

Figure 2. Correlations between mRNA levels of CCR-4 and IFN- $\gamma(\mathbf{A})$, IL-8 and IFN- $\gamma(\mathbf{B})$, CCR-4 and IL-8 (C) in acute skin lesions of patients with AD after UVA1 phototherapy tis patients before and after UVA1 phototherapy. Results were displayed as means and standard deviation. In all calculations, $p<0.05$ was considered statistically significant.

\section{Results}

\section{Correlations before UVA1 irradiation}

Before UVA1 phototherapy, mRNA level of CCR-4 correlated with that of IFN- $\gamma(r=0.61, p<0.05)$ (Figure 1$)$. No significant correlations were found between mRNA level of IL-8 and CCR-4 or between mRNA level of IL-8 and IFN- $\gamma$ before UVA1 phototherapy.

\section{Correlations after UVA1 irradiation}

After UVA1 phototherapy, mRNA level of CCR-4 correlated with that of IFN- $\gamma(r=0.68, p<0.05)$ (Figure 2). A significant correlation was found between mRNA level of IL-8 and IFN- $\gamma(r=0.57, p<0.05)$. After UVA1 photo- 
therapy, mRNA level of CCR-4 correlated also with that of IL-8 $(r=0.54, p<0.05)$.

\section{Impact of UVA1 on mRNA expression}

UVA1 phototherapy resulted in a significant increase in mRNA expression of IL-8 in comparison to the baseline assessment ( $6.74 \pm 3.33$ vs. $8.78 \pm 3.61, p<0.05$, before and after treatment, respectively, $p=0.02$ ) (Figure 3 ).

After UVA1 phototherapy, no significant difference was found with regard to the mRNA expression of CCR-4 $(2.34 \pm 3.67$ vs. $3.34 \pm 3.922$, before and after treatment, respectively) or IFN- $\gamma(12.7 \pm 3.75$ vs. $13.93 \pm 3.0$, before and after treatment, respectively).

\section{SCORAD index and UVA1 phototherapy}

UVA1 phototherapy was associated with a significantly improved SCORAD index (60.16 \pm 14.71 vs. 30.71 \pm 14.41 before and after treatment, respectively, $p<0.001)$ and a significant improvement of the severity of AD (2.68 \pm 0.47 vs. $1.64 \pm 0.56$ before and after treatment, respectively, $p<0.001$ ) (Figure 4).

SCORAD index and severity of AD were not found to correlate with investigated cytokine mRNA expression, neither before nor after UVA1 phototherapy.

\section{Discussion}

The expression of IL-8 mRNA, and the protein itself, by cultured keratinocytes stimulated with Staphylococci [15], indicates that it represents a contributory link in the process of eliciting skin inflammation in response to bacterial colonization or symptomatic skin infection in AD. It is also conceivable that in AD-activated leukocytes in

A

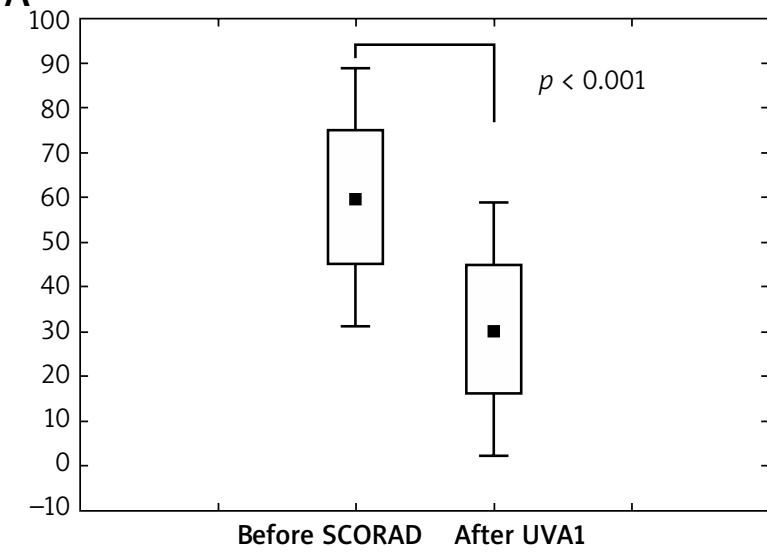

spongiotic or excoriated skin also release IL-8 [10]. Levels of IL-8 in the stratum corneum were found to be associated with the severity of $A D$, particularly with the degree of erythema, edema/papules and excoriation [10]. These observations prompted this investigation of whether the clinical efficacy of UVA1 is related to a decrease in the expression of IL-8 in acute skin lesions in AD. Although UVA1 irradiation resulted in a significant improvement of SCORAD index as well as severity of AD as expected, it

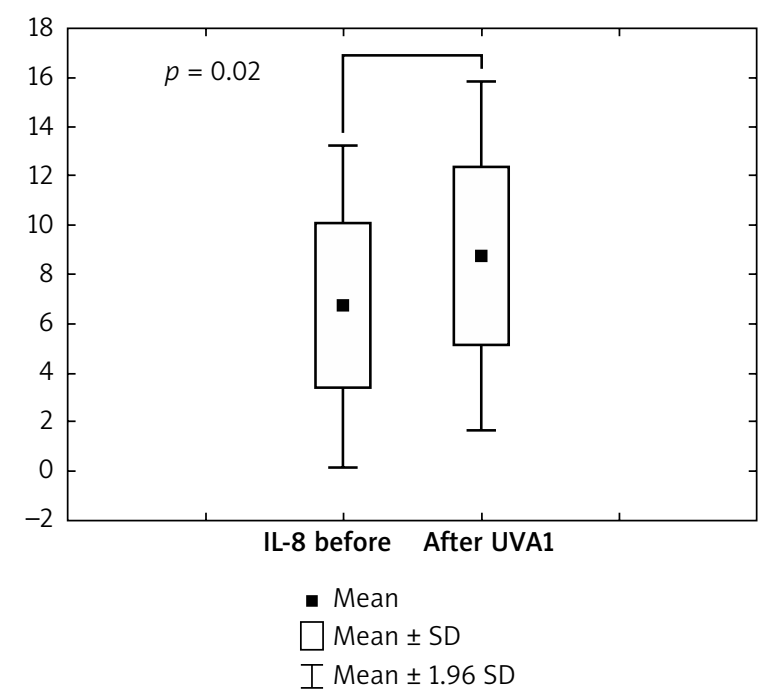

Figure 3. Significant increase in mRNA expression for IL-8 after UVA1 phototherapy. The results of mRNA expression of IL-8 gene are displayed by relative units that represent the differences in cycle threshold numbers between the IL-8 gene and actin beta (ACTB) as an endogenous control

B

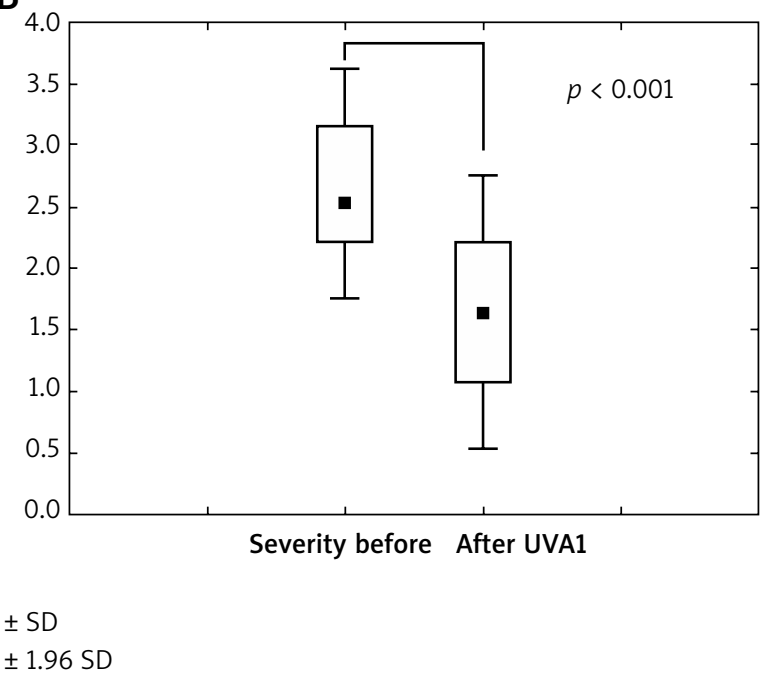

Figure 4. Significant decrease in SCORAD index (A) and severity of AD (B) after UVA1 phototherapy 
was also found to be accompanied with a greater mRNA expression for IL-8. Therefore, it is likely that the efficacy of UVA1 is attributed to its influence on the expression of a number of different molecules, not only IL-8. In interpreting the results of the present study, it is important to consider indirect effects of UVA1 on many other molecules, via downstream mediators of inflammatory pathways in AD. Hence, the results also take into account the effect of UVA1 on the expression of CCR-4 and IFN- $\gamma$ mRNA. CCR4 is a chemokine receptor which is expressed particularly strongly on Th2 lymphocytes, and is involved in the response to macrophage-derived chemokine (MDC) and thymus and activation-regulated chemokine (TARC) [19]. Interestingly, the presence of CCR4(+)CD8(+) T cells predominantly in the CD27(+)CD28(+)CD45RA(-) memory subset was reported [19]. Significant numbers of circulating CCR4(+) memory CD4 lymphocytes were found to express the Th1 cytokine IFN- $\gamma$ after stimulation [19]. The present study reveals correlations between mRNA levels of CCR-4, characteristic of Th2 lymphocytes, and IFN- $\gamma$, a Th1 cytokine, in acute skin lesions of patients with $A D$, both before and after UVA1 phototherapy, which underlines a potential problem in using CCR-4 as a marker of Th2 cells [19]. Instead of the obvious biphasic immune response in $A D$, with a switch from an initial Th2 response, with predominant IL-4, to a Th1 response with its main production of IFN- $\gamma$ in the chronic phase of the disease, patterns of chemokine receptors encountered in vivo can be regulated in a more sophisticated manner [15]. In addition, it seems that interpreting the obtained results as an interplay between Th2 and Th1 cytokine profiles, restricted only to the transition between the initial and the late phase of $A D$, is an over-simplification. Xiao et al. demonstrate that Th2 and Th1 immune responses are not mutually exclusive and that cofactors, spatial separation, such as epidermis vs. dermis, and cell type in particular, play a role in the initiation and perpetuation of inflammation in AD [20]. In a mouse model, production of TARC, a Th2 chemokine, by Langerhans cells was down-regulated by IFN- $\gamma$, a Th1 cytokine, and up-regulated by tumor necrosis factor (TNF)- $\alpha$ and IL-4, aTh2 cytokine [20]. In contrast, HaCaT cells exposed to IL-4, TNF- $\alpha$ and IFN- $\gamma$ characterized significantly decreased supernatant TARC levels compared to those exposed to both TNF- $\alpha$ and IFN- $\gamma$ [21]. These results indicate that the cytokine profile can be regulated in different ways depending on cell type, and that the Th2 and Th1 immune responses are not mutually exclusive. Hence, it is also possible that UVA1 irradiation can exert different effects on the expression of cytokines and chemokines in different cell types in the epidermis and dermis in AD. In this regard, our study has several limitations. Firstly, only full skin biopsies containing both epidermis and dermis were investigated. Secondly, mRNA expression was studied in acute skin lesions and compared only with a constitutively expressed house-keeping gene for beta actin. Undoubtedly, a comparison of mRNA expression levels in affected and unaffected skin, together with skin samples from healthy control individuals, would provide more comprehensive data. Thirdly, an investigation of selected molecules additionally at the protein level would also supply more convincing evidence. However, this will have to await future studies. Our intention was to investigate and to confirm, firstly, that IL-8, IFN- $\gamma$, and CCR-4 are expressed, and that secondly, CCR-4 is expressed in parallel with IFN- $\gamma$ in acute skin lesions of patients with AD both before and after UVA1 phototherapy. Another key conclusion is the fact that UVA1 significantly improves SCORAD index, lessens the severity of AD and increases the expression of IL-8, with no direct effects on other studied molecules. However, it is likely that UVA1 can influence inflammatory pathways in AD via indirect effects by other mediators. Undoubtedly, the results of RT-PCR are comparable and reproducible, and so further studies conducted on larger populations of patients with AD from different sites are required. This reproducibility may allow future conclusions to be drawn from a more comprehensive evidence base.

\section{Acknowledgments}

This study was supported by a grant for scientific purposes (No. 502-03/1-152-01/502-14-255) from the Medical University of Lodz, Poland.

\section{Conflict of interest}

The authors declare no conflict of interest.

\section{References}

1. Krutmann J, Czech W, Diepgen T, et al. High-dose UVA1 therapy in the treatment of patients with atopic dermatitis. J Am Acad Dermatol 1992; 26: 225-30.

2. Sándor N, Pap D, Prechl J, et al. A novel, complement-mediated way to enhance the interplay between macrophages, dendritic cells and T lymphocytes. Mol Immunol 2009; 47: 438-48.

3. Breuckmann F, von Kobyletzki G, Avermaete A, et al. Mononuclear cells in atopic dermatitis in vivo: immunomodulation of the cutaneous infiltrate by medium-dose UVA1 phototherapy. Eur J Med Res 2002; 7: 315-22.

4. Campbell JJ, Haraldsen G, Pan J, et al. The chemokine receptor CCR4 in vascular recognition by cutaneous but not intestinal memory T cells. Nature 1999; 400: 776-80.

5. Gambichler T, Kreuter A, Tomi NS, et al. Gene expression of cytokines in atopic eczema before and after ultraviolet A1 phototherapy. Br J Dermatol 2008; 158: 1117-20.

6. Grabbe J, Welker P, Humke S, et al. High-dose ultraviolet A1 (UVA1), but not UVA/UVB therapy, decreases IgE-binding cells in lesional skin of patients with atopic eczema. J Invest Dermatol 1996; 107: 419-22.

7. Kimata H, Lindley I. Detection of plasma interleukin-8 in atopic dermatitis. Arch Dis Child 1994; 70: 119-22.

8. Kreuter A, Hyun J, Skrygan M, et al. Ultraviolet A1-induced downregulation of human beta-defensins and interleukin- 6 
and interleukin-8 correlates with clinical improvement in localized scleroderma. Br J Dermatol 2006; 155: 600-7.

9. Mukaida N. Interleukin-8: an expanding universe beyond neutrophil chemotaxis and activation. Int J Hematol 2000; 72: 391-8.

10. Amarbayasgalan T, Takahashi H, Dekio I, Morita E. Interleukin-8 content in the stratum corneum as an indicator of the severity of inflammation in the lesions of atopic dermatitis. Int Arch Allergy Immunol 2013; 160: 63-74.

11. Larsen CG, Anderson AO, Oppenheim JJ, Matsushima K. Production of interleukin-8 by human dermal fibroblasts and keratinocytes in response to interleukin-1 or tumour necrosis factor. Immunology 1989; 68: 31-6.

12. Möller A, Lippert U, Lessmann D, et al. Human mast cells produce IL-8. J Immunol 1993; 151: 3261-6.

13. Hanifin JM, Rajka G. Diagnostic features of atopic dermatitis. Acta Derm Venereol 1980; 90: 44-7.

14. Ring J, Alomar A, Bieber T, et al. Guidelines for treatment of atopic eczema (atopic dermatitis). Part II. J Eur Acad Dermatol Venereol 2012; 26: 1176-93.

15. Thepen T, Langeveld-Wildschut EG, Bihari IC, et al. Biphasic response against aeroallergen in atopic dermatitis showing a switch from an initial Th2 response to a Th1 response in situ: an immunocytochemical study. J Allergy Clin Immunol 1996; 97: 828-37.

16. Werfel T, Morita A, Grewe M, et al. Allergen specificity of skin-infiltrating $T$ cells is not restricted to a type-2 cytokine pattern in chronic skin lesions of atopic dermatitis. J Invest Dermatol 1996; 107: 871-6.

17. Kunz B, Oranje AP, Labrèze L, et al. Clinical validation and guidelines for the SCORAD index: consensus report of the European Task Force on Atopic Dermatitis. Dermatology 1997; 195: 10-9.

18. Sasaki T, Kano R, Sato H, et al. Effects of staphylococci on cytokine production from human keratinocytes. Br J Dermatol 2003; 148: 46-50.

19. Kondo T, Takiguchi M. Human memory CCR4+CD8+ T cell subset has the ability to produce multiple cytokines. Int Immunol 2009; 21: 523-32.

20. Xiao T, Fujita H, Saeki H, et al. Thymus and activation-regulated chemokine (TARC/CCL17) produced by mouse epidermal Langerhans cells is upregulated by TNF-alpha and IL-4 and downregulated by IFN-gamma. Cytokine 2003; 23: 126-32.

21. Kakinuma T, Nakamura K, Wakugawa M, et al. IL-4, but not IL-13, modulates TARC (thymus and activation-regulated chemokine)/CCL17 and IP-10 (interferon-induced protein of $10 \mathrm{kDA}$ )/CXCL10 release by TNF-alpha and IFN-gamma in HaCaT cell line. Cytokine 2002; 20: 1-6. 